\title{
Spectral Properties for the Magnetization Integral Operator
}

\author{
By Mark J. Friedman* and Joseph E. Pasciak**
}

\begin{abstract}
We analyze the spectrum of a certain singular integral operator on the space $\left(L^{2}(\Omega)\right)^{3}$ where $\Omega$ is contained in three dimensional Euclidean space and has a Lipschitz continuous boundary. This operator arises in the integral formulation of the magnetostatic field problem. We decompose $\left(L^{2}(\Omega)\right)^{3}$ into invariant subspaces: in one where the operator is the zero map; in one, the identity map; and in one where the operator is positive definite and bounded. These results give rise to the formulation of new efficient numerical techniques for approximating nonlinear magnetostatic field problems [5], [6], [12].
\end{abstract}

1. Introduction. In this paper we analyze the spectrum of the singular integral operator

$$
A w=\nabla T w
$$

where

$$
(T w)(x)=\frac{1}{4 \pi} \int_{\Omega} w(y) \cdot \nabla_{y}\left(\frac{1}{r}\right) d y \quad \text { and } \quad r=|x-y|
$$

defined on $w \in\left(L^{2}(\Omega)\right)^{3}$ for bounded domains $\Omega$ contained in $R^{3}$. The operator $A$ is used in integral formulations of the magnetostatic field problem and their discretization [1], [3], [11]. Applications of the results given in this paper lead to new efficient numerical procedures [5], [6], [12] for approximating nonlinear magnetostatic field problems.

The spectrum of the operator (1.1) was first analyzed in [4] for smooth simply connected domains by the methods of classical potential theory. In this paper we extend these results to the case of domains with Lipschitz continuous boundaries. In contrast, our analysis is based on deriving an equivalent formulation of the operator as an elliptic boundary value problem. The desired results are obtained by developing the appropriate properties of the boundary value problem.

The outline of the remainder of the paper is as follows. In Section 2, we introduce some notation and state some preliminary results. In Section 3, we state and prove

Received March 17, 1983; revised January 12, 1984.

1980 Mathematics Subject Classification. Primary 45E10; Secondary 35 J60.

* Work supported by Cornell University and Iowa State University.

** The submitted manuscript has been authored under contract DE-AC02-76CH00016 with the U. S. Department of Energy. Accordingly, the U. S. government retains a nonexclusive, royalty-free license to publish or reproduce the published form of this contribution, or allow others to do so, for U. S. Government purposes. 
the main results of the paper (Theorem 1 and Corollary 1) which describe the spectral decomposition of the operator $A$.

2. Preliminaries and Notation. Let $\Omega$ denote a bounded domain in three dimensional Euclidean space $R^{3}$ with Lipschitz continuous boundary $\Gamma$. Sobolev spaces on $\Omega$ and $\Gamma$ of order $s$ will be denoted $H^{s}(\Omega)$ and $H^{s}(\Gamma)$ with corresponding norms $\|\cdot\|_{H^{s}(\Omega)}$ and $|\cdot|_{H^{s}(\Gamma)}$, respectively, [7], [9]. For negative $s$, the Sobolev spaces are defined by duality. Let $\mathscr{D}(\Omega)$ be the space of infinitely differentiable functions with support contained in $\Omega$ and $\mathscr{D}^{\prime}(\Omega)$ denote the space of Schwartz distributions on $\Omega$ [14]. $C^{\infty}(\bar{\Omega})$ denotes the space of infinitely differentiable functions on $\bar{\Omega}$ and is dense in $H^{s}(\Omega)$ for any s. $H_{0}^{s}(\Omega)$ is defined to be the completion of $\mathscr{D}(\Omega)$ in the $H^{s}(\Omega)$ norm.

The notation $\underline{H}$ will denote the product space $H^{3}$ which has components in a space $H$. When $H$ is a Hilbert space, $\underline{H}$ inherits the obvious norms and inner products.

Let $(\cdot, \cdot)_{\Omega}$ denote the $L^{2}$ inner product on $\Omega$ given by

$$
(u, v)_{\Omega} \equiv \int_{\Omega} u v d x
$$

For vector-valued functions $u=\left(u_{1}, u_{2}, u_{3}\right)$ and $v=\left(v_{1}, v_{2}, v_{3}\right)$, (2.1) will be replaced by

$$
(u, v)_{\Omega}=\int_{\Omega} u \cdot v d x .
$$

The Dirichlet inner product on $\Omega$ is given by

$$
D_{\Omega}(u, v) \equiv(\nabla u, \nabla v)_{\Omega} .
$$

The Lipschitz continuity assumption on $\Gamma$ implies that the exterior normal $n=\left(n_{1}, n_{2}, n_{3}\right)$ on $\Gamma$ exists almost everywhere [9]. In addition there are trace and extension operators as described by the following lemma, which may be found in [9].

LEMMA 1 . The trace operator (denoted by $T_{\Omega}$ ) extends continuously from $\mathscr{D}(\bar{\Omega})$ to an operator from $H^{1}(\Omega)$ onto $H^{1 / 2}(\Gamma)$.

LEMMA 2. There exists a bounded extension operator $E_{\Omega}$ from $H^{1 / 2}(\Gamma)$ into $H^{1}(\Omega)$ satisfying

$$
T_{\Omega} \circ E_{\Omega}=I \text { on } H^{1 / 2}(\Gamma)
$$

Our results for the singular integral operator will be stated for a fixed bounded domain $\Omega$ with Lipschitz continuous boundary $\Gamma$. We shall denote the $L^{2}$ inner product on $\Gamma$ by $\langle\cdot, \cdot\rangle$. In addition $\langle\cdot, \cdot\rangle$ shall be used to denote the duality between $H^{s}(\Gamma)$ and $H^{-s}(\Gamma)$.

We shall need some auxiliary subspaces of $L^{2}(\Omega)$. Let

$$
K \equiv\left\{\nabla \phi \mid \phi \in H^{1}(\Omega)\right\}
$$

Define

$$
\mathscr{N}_{\Omega} \equiv\left\{\phi \in H^{1}(\Omega) \mid \nabla \phi=0 \text { in } \Omega\right\}
$$


Note that the functions in $\mathcal{N}_{\Omega}$ are constant on the components of $\Omega$ and $\Omega$ has only a finite number of components. Set

$$
\stackrel{\circ}{H}^{1}(\Omega) \equiv\left\{\phi \in H^{1}(\Omega) \mid(\phi, \psi)_{\Omega}=0 \text { for all } \psi \in \mathscr{N}_{\Omega}\right\} .
$$

$\stackrel{H}{H}^{1}(\Omega)$ is obviously a closed subspace of $H^{1}(\Omega)$. A standard argument used in the proof of the Poincaré-Friedrichs inequality [2] gives that for some $c>0$

$$
\|\phi\|_{H^{1}(\Omega)}^{2} \leqslant c D_{\Omega}(\phi, \phi) \text { for } \phi \in \stackrel{\circ}{H}^{1}(\Omega)
$$

and hence $\left\{D_{\Omega}(\cdot, \cdot)\right\}^{1 / 2}$ is a norm on $\stackrel{\circ}{H}^{1}(\Omega)$ which is equivalent to the usual Sobolev norm.

Note that $K$ obviously coincides with

$$
\left\{\nabla \phi \mid \phi \in \stackrel{\circ}{H}^{1}(\Omega)\right\}
$$

and it is a straightforward consequence of (2.2) that $K$ is closed in $L^{2}(\Omega)$.

Let

$$
K_{0} \equiv\left\{\nabla \phi \mid \phi \in H_{0}^{1}(\Omega)\right\} .
$$

Then, arguments similar to those given above imply that $K_{0}$ is a closed subspace of $L^{2}(\Omega)$. The orthogonal complement of $K_{0}$ in $K$ will be denoted $K_{H}$ and the orthogonal complement of $K$ in $L^{2}(\Omega)$ will be denoted by $N$. The following lemma was given by Temam [13].

LEMMA 3. $N$ is the completion in $L^{2}(\Omega)$ of

$$
\{u \in \underline{\mathscr{D}(\Omega)} \mid \operatorname{div} u=0\} \text {. }
$$

3. The Spectral Properties of $A$. Let $w$ be a Lipschitz continuous vector field defined on $\Omega$. The kernel of the integral operator $T$ is weakly singular and it is shown in [8] that the partial derivatives of $T w$ exist. Furthermore, the map $w$ to $\partial T w / \partial x_{i}$ extends continuously to a bounded operator from $L^{p}(\Omega)$ into $L^{p}(\Omega)$ for $p>1$. The map $T$ is thus a bounded map from $L^{2}(\Omega)$ to $H^{1}(\Omega)$.

The goal of this paper is to prove the following theorem and its corollary.

THEOREM 1. The operator $A$ is a bounded selfadjoint map on $L^{2}(\Omega)$ and satisfies

(i) $\operatorname{Ker} A=N$.

(ii) $A$ is the identity when restricted to $K_{0}$.

(iii) $K_{H}$ is an invariant subspace of $A$.

(iv) The spectrum of $A$ on $K_{H}$ is contained in the interval $\left[\lambda_{0}, \Lambda_{0}\right]$ where $0<\lambda_{0} \leqslant$ $\Lambda_{0} \leqslant 1$.

Corollary 1. $\Lambda_{0}$ in Theorem 1 can be taken less than 1 if and only if the complement of $\Omega$ has no bounded components.

We shall need additional notation and lemmas for the proof of Theorem 1. Let $\Omega^{\prime}$ be an arbitrary domain in $R^{3}$. As in [10], we consider the spaces $W_{0}^{1}\left(\Omega^{\prime}\right)$ defined by

$$
W_{0}^{1}\left(\Omega^{\prime}\right) \equiv\left\{\phi \in \mathscr{D}^{\prime}\left(\Omega^{\prime}\right) \mid \nabla \phi \in \underline{L^{2}\left(\Omega^{\prime}\right)} \text { and } \phi /(1+r) \in L^{2}\left(\Omega^{\prime}\right)\right\}
$$

where $r$ measures the distance to the origin. Then $W_{0}^{1}\left(\Omega^{\prime}\right)$ has the natural norm

$$
\|\phi\|_{W_{0}^{1}\left(\Omega^{\prime}\right)}=\left\{\|\nabla \phi\|_{L^{2}\left(\Omega^{\prime}\right)}^{2}+\|\phi /(1+r)\|_{L^{2}\left(\Omega^{\prime}\right)}^{2}\right\}^{1 / 2} .
$$

The following lemma is given in [10]. 
LEMMA 4. If $\Omega^{\prime}$ has no bounded components then (3.1) is equivalent to the norm $\left\{D_{\Omega}(u, u)\right\}^{1 / 2}$ for $u$ in $W_{0}^{1}\left(\Omega^{\prime}\right)$.

Let $\Omega_{c}$ denote the interior of the complement of the region $\Omega$. For any bounded domain $\Omega^{\prime}$ contained in $\Omega_{c}$, any function in $V_{0}^{1}\left(\Omega_{c}\right)$, when restricted to $\Omega^{\prime}$, is in $H^{1}\left(\Omega^{\prime}\right)$ and thus it is a straightforward consequence of Lemmas 1 and 2 that there exist appropriately bounded trace and extension operators $T_{\Omega_{c}}$ and $E_{\Omega_{c}}$ between $W_{0}^{1}\left(\Omega_{c}\right)$ and $H^{1 / 2}(\Gamma)$. Let

$$
\mathscr{N}_{\Omega_{c}} \equiv\left\{\phi \in W_{0}^{1}\left(\Omega_{c}\right) \mid \nabla \phi=0 \text { on } \Omega_{c}\right\} .
$$

Note that functions in $\mathscr{N}_{\Omega_{c}}$ are constant on the bounded components of $\Omega_{c}$ and zero on the unbounded component of $\Omega_{c}$. We define

$$
\stackrel{\circ}{W}_{0}^{1}\left(\Omega_{c}\right) \equiv\left\{\phi \in W_{0}^{1}\left(\Omega_{c}\right) \mid(\phi, \psi)_{\Omega_{c}}=0 \text { for all } \psi \in \mathscr{N}_{\Omega_{c}}\right\} .
$$

Then Lemma 4 and (2.2) imply that $\left\{D_{\Omega_{c}}(\cdot, \cdot)\right\}^{1 / 2}$ is a norm equivalent to (3.1) on $\stackrel{\circ}{W}_{0}^{1}\left(\Omega_{c}\right)$. Let $W_{0,0}^{1}\left(\Omega_{c}\right)$ be defined by

$$
W_{0,0}^{1}\left(\Omega_{c}\right) \equiv\left\{\psi \in W_{0}^{1}\left(\Omega_{c}\right) \mid T_{\Omega_{c}}(\psi)=0\right\},
$$

and

$$
K_{H}\left(\Omega_{c}\right)=\left\{\nabla \psi \mid \psi \in W_{0}^{1}\left(\Omega_{c}\right) \text { and } D_{\Omega_{c}}(\psi, \phi)=0 \text { for all } \phi \in W_{0,0}^{1}\left(\Omega_{c}\right)\right\} .
$$

We shall use the following lemmas in the proof of Theorem 1.

LeMma 5. For $\phi \in H^{1 / 2}(\Gamma)$ there exists a unique extension $h_{\phi}^{\Omega}$ in $H^{1}(\Omega)$ satisfying

$$
D_{\Omega}\left(h_{\phi}^{\Omega}, \psi\right)=0 \quad \text { for all } \psi \in H_{0}^{1}(\Omega) \text {. }
$$

The above statement holds with $\Omega, H^{1}(\Omega)$ and $H_{0}^{1}(\Omega)$ replaced by $\Omega_{c}, W_{0}^{1}\left(\Omega_{c}\right)$ and $W_{0,0}^{1}\left(\Omega_{c}\right)$.

Remark. $h_{\phi}^{\Omega}$ (resp. $h_{\phi}^{\Omega_{c}}$ ) is just the harmonic extension of $\phi$ into $\Omega$ (resp. $\Omega_{c}$ ).

Let $\mathscr{N}_{\Gamma} \equiv\left\{T_{\Omega}(\psi) \mid \psi \in \mathscr{N}_{\Omega}\right\}$ and define

$$
H^{-1 / 2}(\Gamma) / \mathscr{N}_{\Gamma} \equiv\left\{\phi \in H^{-1 / 2}(\Gamma) \mid\langle\phi, \psi\rangle=0 \text { for all } \psi \in \mathscr{N}_{\Gamma}\right\} .
$$

LEMMA 6. For a function $\sigma$ in $H^{-1 / 2}(\Gamma) / \mathcal{N}_{\Gamma}$ there exists a unique function $\psi$ in $\stackrel{\circ}{H}^{1}(\Omega)$ satisfying

$$
D_{\Omega}(\psi, \theta)=\langle\sigma, \theta\rangle \text { for all } \theta \in H^{1}(\Omega) .
$$

Furthermore, the map $\sigma \rightarrow \nabla \psi$ is a homeomorphism of $H^{-1 / 2}(\Gamma) / \mathscr{N}_{\Gamma}$ onto $K_{H} \cdot A$ similar result holds with $\mathscr{N}_{\Gamma}, \stackrel{\circ}{H}^{1}(\Omega), H^{1}(\Omega)$ and $K_{H}$ replaced by $\mathscr{N}_{\Gamma_{c}}, \stackrel{\circ}{W}_{0}^{1}\left(\Omega_{c}\right)$, $W_{0}^{1}\left(\Omega_{c}\right)$ and $K_{H}\left(\Omega_{c}\right)$ respectively.

Remark. $\sigma$ is the generalized outward normal derivative of $\psi$ on $\Gamma$.

Lemma 7. Let $w$ be in $L^{2}(\Omega)$. Then $u=T w$ is the unique function in $W_{0}^{1}\left(R^{3}\right)$ satisfying

$$
D_{R^{3}}(u, \phi)=(w, \nabla \phi)_{\Omega} \quad \text { for all } \phi \in W_{0}^{1}\left(R^{3}\right) .
$$

We postpone the proof of the last three lemmas until after the proof of the theorem and its corollary. 
Proof of Theorem 1. Lemma 7 and the definition of $A$ imply that $A w=\nabla u$ where $u$ is the solution of (3.3). Substituting $\phi=T v$ in (3.3) gives

$$
(A w, A v)_{R^{3}}=(w, A v)_{\Omega}
$$

and implies that $A$ is symmetric and bounded, hence selfadjoint.

If $w$ is in $K_{0}$, then $w=\nabla v$ for some $v \in H_{0}^{1}(\Omega)$ and $v$ extended by zero is in $W_{0}^{1}\left(R^{3}\right)$. Hence, $v$ extended by zero is the solution of (3.3) and thus $A w=\nabla v=w$ which proves (ii).

We next show that there exists $\lambda_{0}>0$ satisfying

$$
\lambda_{0}\|w\|_{\underline{L^{2}(\Omega)}} \leqslant\|A w\|_{\underline{L^{2}(\Omega)}} \text { for all } w \in K_{H} .
$$

Let $w$ be in $K_{H}$ then $w=\nabla \psi$ for some $\psi \in H^{1}(\Omega)$. Let $\sigma_{w}$ be the distribution guaranteed by Lemma 6 satisfying

$$
D_{\Omega}(\psi, \phi)=\left\langle\sigma_{w}, \phi\right\rangle \text { for all } \phi \in H^{1}(\Omega) .
$$

If $u$ is the solution of (3.3), then $\nabla u$ is in $K_{H}\left(\Omega_{c}\right)$. Let $\sigma_{u}$ be the corresponding distribution satisfying

$$
D_{\Omega_{c}}(u, \phi)=\left\langle\sigma_{u}, \phi\right\rangle \text { for all } \phi \in W_{0}^{1}\left(\Omega_{c}\right) .
$$

Let $\phi$ be an arbitrary function in $H^{1 / 2}(\Gamma)$ and extend $\phi$ to $R^{3}$ by

$$
\bar{\phi} \equiv \begin{cases}E_{\Omega}(\phi) & \text { on } \Omega, \\ E_{\Omega_{c}}(\phi) & \text { on } \Omega_{c} .\end{cases}
$$

Then $\bar{\phi}$ is in $W_{0}^{1}\left(R^{3}\right)$ and using (3.3), (3.6), and (3.7)

$$
\begin{aligned}
\left|\left\langle\sigma_{w}, \phi\right\rangle\right| & =\left|(w, \nabla \bar{\phi})_{\Omega}\right|=\left|D_{\Omega}(u, \bar{\phi})+\left\langle\sigma_{u}, \phi\right\rangle\right| \\
& \leqslant c\left\{\|\nabla u\|_{L^{2}(\Omega)}+\left|\sigma_{u}\right|_{H^{-1 / 2}(\Gamma)}\right\}|\phi|_{H^{1 / 2}(\Gamma)} \\
& \leqslant c\|A w\|_{\underline{L^{2}\left(R^{3}\right)}}|\phi|_{H^{1 / 2}(\Gamma)} .
\end{aligned}
$$

Thus by Lemma 6 and the definition of the norm in $H^{-1 / 2}(\Gamma)$ we have

$$
\|w\|_{\underline{L^{2}(\Omega)}}^{2} \leqslant c\left|\sigma_{w^{\prime}}\right|_{H^{-1 / 2}(\Gamma)}^{2} \leqslant \frac{1}{\lambda_{0}}\|A w\|_{\underline{L^{2}\left(R^{3}\right)}}^{2} .
$$

Thus (3.5) follows from (3.4) and the Schwarz inequality.

By (3.3) and the definition of $N, N$ is contained in $\operatorname{Ker} A$ and (i) follows from (ii) and (3.5). We also note that (3.4) with $v=w$ and the Schwarz inequality imply that

$$
\|A w\|_{L^{2}(\Omega)} \leqslant\|w\|_{L^{2}(\Omega)}
$$

and thus the theorem will follow once (iii) is verified.

From the definition $A w=\nabla T w$, the range of $A$ is contained in $K$. Let $w$ be in $K_{H}$, $u$ solve (3.3) and $\sigma_{u}$ be defined as above. If $v=\nabla \psi$ is in $K_{0}$ then Lemma 7 implies that

$$
0=(w, \nabla \psi)_{\Omega}=D_{\Omega}(u, \psi)+\left\langle\sigma_{u}, \psi\right\rangle=(\nabla u, \nabla \psi)_{\Omega} .
$$

Thus $A w=\nabla u$ is in $K_{H}$ and the proof of the theorem is complete.

Proof of Corollary 1. Assume that $\Omega_{c}$ has no bounded components. Let $w$ be in $K_{H}$ and let $u$ and $\sigma_{u}$ be as in the proof of Theorem 1. Then by (3.3), Lemmas 1 and 4

$$
(w, A w)_{\Omega}=\|A w\|_{\underline{L^{2}(\Omega)}}^{2}+\|\nabla u\|_{\underline{L^{2}\left(\Omega_{c}\right)}}^{2} \geqslant\|A w\|_{\underline{L^{2}(\Omega)}}^{2}+c|u|_{H^{1 / 2}(\Gamma)}^{2} .
$$


Now since $u=h_{u}^{\Omega}$, Lemma 5 implies

$$
\|A w\|_{\underline{L^{2}(\Omega)}}=\|\nabla u\|_{\underline{L^{2}(\Omega)}} \leqslant c|u|_{H^{1 / 2}(.,}
$$

thus

$$
(w, A w)_{\Omega} \geqslant(1+c)\|A w\|_{\underline{L^{2}(\Omega)}}^{2}
$$

with $c>0$. This proves the first part of the corollary.

Suppose $\Omega_{c}$ has a bounded component which we will denote $\Omega_{c}^{b}$. Define $\phi$ on $\Gamma$ by

$$
\phi(x) \equiv \begin{cases}1 & \text { if } x \in \partial \Omega_{c}^{b}, \\ 0 & \text { if } x \in \Gamma-\partial \Omega_{c}^{b} .\end{cases}
$$

Define $w=\nabla h_{\phi}^{\Omega}$; then by Lemma $5, w$ is in $K_{H}$. Furthermore, it is easily seen that

$$
u= \begin{cases}h_{\phi}^{\Omega} & \text { on } \Omega, \\ h_{\phi}^{\Omega_{c}} & \text { on } \Omega_{c}\end{cases}
$$

is constant on the components of $\Omega_{c}$ and hence is the solution of (3.3). Thus $A w=w$ and the corollary follows.

We shall only sketch the proofs of Lemmas 5 and 6 since the lemmas correspond to results which are well known in the case of smooth domains.

Proof of Lemma 5. We prove Lemma 5 for the domain $\Omega$. Given $\phi$ in $H^{1 / 2}(\Gamma)$ define $\psi=E_{\Omega}(\phi)$ and let $v$ be the unique function in $H_{0}^{1}(\Omega)$ satisfying

$$
D_{\Omega}(v+\psi, \theta)=0 \text { for all } \theta \in H_{0}^{1}(\Omega) .
$$

Then $h_{\phi}^{\Omega}=v+\psi$ has the desired properties. The uniqueness of the extension $h_{\phi}^{\Omega}$ is a consequence of the fact that

$$
H_{0}^{1}(\Omega)=\left\{\phi \in H^{1}(\Omega) \mid T_{\Omega} \phi=0\right\} .
$$

Proof of Lemma 6. We prove Lemma 6 for $\Omega$, the proof for $\Omega_{c}$ is similar. Let $\sigma$ be in $H^{-1 / 2}(\Gamma) / \mathscr{N}_{\Gamma}$; then the map $\theta \rightarrow\langle\sigma, \theta\rangle$ is a bounded linear functional on $\stackrel{\circ}{H}^{1}(\Omega)$. Since $\|\nabla \phi\|_{L^{2}(\Omega)}$ is a norm on $\stackrel{\circ}{H}^{1}(\Omega)$, the Riesz Representation Theorem guarantees that there exists $\psi$ in $\stackrel{\circ}{H}^{1}(\Omega)$ satisfying (3.2) for all $\theta$ in $\stackrel{H}{H}^{1}(\Omega)$. From the definition of $H^{-1 / 2}(\Gamma) / \mathscr{N}_{\Gamma}$, this also implies that $\psi$ satisfies (3.2) for all functions $\theta$ in $H^{1}(\Omega)$. The map $S(\sigma) \equiv \nabla \psi$ is clearly bounded from $H^{-1 / 2}(\Gamma) / \mathscr{N}_{\Gamma}$ into $K_{H}$.

Given $w=\nabla \phi$ in $K_{H}$ we have by Lemma 5

$$
\left|D_{\Omega}\left(\psi, h_{\psi}^{\Omega}\right)\right| \leqslant C\|\nabla \psi\|_{L^{2}(\Omega)}|\psi|_{H^{1 / 2}(\Gamma)} \text { for all } \psi \in H^{1 / 2}(\Gamma) .
$$

Thus there exists a unique distribution $\sigma$ in $H^{-1 / 2}(\Gamma)$ satisfying

$$
\langle\sigma, \phi\rangle=D_{\Omega}\left(\psi, h_{\phi}^{\Omega}\right) \quad \text { for all } \phi \in H^{1 / 2}(\Gamma) .
$$

One then argues that the map $w \rightarrow \sigma$ is a bounded map of $K_{H}$ into $H^{-1 / 2}(\Gamma) / \mathscr{N}_{\Gamma}$ and $S(\sigma)=w$. Hence $S$ is a homeomorphism of $H^{-1 / 2}(\Gamma) / \mathscr{N}_{\Gamma}$ onto $K_{H}$.

Proof of Lemma 7. Let $w$ be in $L^{2}(\Omega)$. Choose $\left\{w_{i}\right\}_{i=1}^{\infty}$ in $\mathscr{D}(\Omega)$ with $w_{i}$ converging to $w$ in $L^{2}(\Omega)$. Clearly the functional $\phi \rightarrow(w, \nabla \phi)_{\Omega}$ is bounded on $W_{0}^{1}\left(R^{3}\right)$. Thus by Lemma 4 there exists a unique function $u_{i} \in W_{0}^{1}\left(R^{3}\right)$ satisfying

$$
D_{R^{3}}\left(u_{i}, \phi\right)=\left(w_{i}, \nabla \phi\right)_{\Omega} \text { for all } \phi \in W_{0}^{1}\left(R^{3}\right) \text {. }
$$


Clearly $u_{i}$ converges to the solution $u$ of (3.3) in $W_{0}^{1}\left(R^{3}\right)$. It is also clear from classical potential theory that

$$
u_{i}=\frac{1}{4 \pi} \int_{R^{3}} w_{i} \cdot \nabla_{y}\left(\frac{1}{r}\right) d y=\frac{1}{4 \pi} \int_{\Omega} w_{i} \cdot \nabla_{y}\left(\frac{1}{r}\right) d y .
$$

Thus, $T w_{i}=u_{i}$ and the lemma follows by density.

Department of Mathematics

Iowa State University

Ames, Iowa 50011

Applied Mathematics Department

Brookhaven National Laboratory

Upton, New York 11973

1. A. G. Armstrong, A. M. Collie, C. J. Diserens, N. J. Newman, M. Simkin \& C. W. Trowbridge, New Developments in the Magnet Design Program GFUN, Rutherford Laboratory Report No. RL-5060.

2. P. G. Ciaruet, The Finite Element Method for Elliptic Problems, Vol. 4, North-Holland, Amsterdam, 1978.

3. M. J. Friedman, "Mathematical study of the nonlinear singular integral magnetic field equation I," SIAM J. Appl. Math., v. 39, 1980, pp. 14-20.

4. M. J. Friedman, "Mathematical study of the nonlinear singular integral magnetic field equation III," SIAM J. Math. Anal., v. 12, 1981, pp. 536-540.

5. M. J. Friedman \& J. S. Colonias, "On the coupled differential-integral equations for the solution of the general magnetostatic problem," IEEE Trans. Mag., v. Mag-18, No. 2, March 1982, pp. 336-339.

6. M. J. Friedman, "Finite element formulation of the general magnetostatic problem in the space of solenoidal vector functions," Math. Comp., v. 43, 1984, pp. 415-431.

7. J. L. Lions \& E. MAgenes, Nonhomogeneous Boundary Value Problems and Applications, Vol. 1, Springer-Verlag, New York, 1972.

8. S. G. Mikhuin, Multidimensional Singular Integrals and Integral Equations, Pergamon Press, Oxford, New York, 1965.

9. J. NeCAS, Les Méthodes Directes en Théorie des Équations Elliptiques, Masson, Paris, 1967.

10. J. C. Nedelec, Approximation des Équations Intégrales en Mécanique et en Physique, Centre de Mathématiques Appliquées, École Polytechnique, Palaiseau, 1977.

11. J. PASCIAK, "An iterative algorithm for the volume integral method for magnetostatics problems," Comput. Math. Appl., v. 8, 1982, pp. 283-290.

12. J. PASCiaK, "A new scalar potential formulation of the magnetostatic field problem," Math. Comp., v. 43,1984 , pp. $433-445$.

13. R. Tемам, On the Theory and Numerical Analysis of the Navier-Stokes Equations, North-Holland, Amsterdam, 1977.

14. V. S. Vladimirov, Equations of Mathematical Physics, Marcel Dekker, New York, 1971. 